\title{
Chronic dietary intake of $\alpha$-linolenic acid does not replicate the effects of DHA on passive properties of entorhinal cortex neurons
}

\author{
Dany Arsenault ${ }^{1,2}$, Carl Julien ${ }^{1,2}$ and Frédéric Calon ${ }^{1,2 *}$ \\ ${ }^{1}$ Faculté de pharmacie, Université Laval, Québec, QC, Canada \\ ${ }^{2}$ Neurosciences Axis, Centre Hospitalier de l'Université Laval (CHUL) Research Center, CHUQ, 2705, Laurier Boulevard, \\ Room T2-05, Québec, QC, Canada G1V 4 G2
}

(Submitted 4 March 2011 - Final revision received 24 June 2011 - Accepted 27 June 2011 - First published online 19 August 2011)

\begin{abstract}
$n$-3 PUFA are receiving growing attention for their therapeutic potential in central nervous system (CNS) disorders. We have recently shown that long-term treatment with DHA alters the physiology of entorhinal cortex (EC) neurons. In the present study, we investigated by patchclamp the effect of another major dietary $n$-3 PUFA, $\alpha$-linolenic acid (LNA), on the intrinsic properties of EC neurons. Mice were chronically exposed to isoenergetic diets deficient in $n-3$ PUFA or enriched in either DHA or LNA on an equimolar basis. GC analyses revealed an increase in DHA (34\%) and a decrease in arachidonic acid (AA, - 23\%) in brain fatty acid concentrations after consumption of the DHA-enriched diet. Dietary intake of LNA similarly affected brain fatty acid profiles, but at a lower magnitude (DHA: 23\%, AA: $-13 \%)$. Compared to the $n$-3 PUFA-deficient diet, consumption of DHA, but not LNA, induced membrane hyperpolarisation ( -60 to $-70 \mathrm{mV})$, increased cellular capacitance $(32 \%)$ and spontaneous excitatory postsynaptic current frequency (50\%). We propose that the inefficiency of LNA to modulate cellular capacitance was related to its inability to increase the brain DHA:AA ratio over the threshold necessary to up-regulate syntaxin-3 (46\%) and translocate drebrin (40\% membrane:cytosol ratio). In summary, our present study shows that the increase in brain DHA content following chronic administration of LNA was not sufficient to alter the passive and synaptic properties of EC neurons, compared to direct dietary intake of DHA. These diverging results have important implications for the therapeutic use of $n-3$ PUFA in CNS disease, favouring the use of preformed DHA.
\end{abstract}

Key words: DHA: Linolenic acid: Electrophysiology: Diets: Mice: Intrinsic properties: Entorhinal cortex

n-3 PUFA are essential dietary lipids receiving considerable attention as either potential nutraceuticals or protective factors for prevalent diseases. Physiologically relevant $n-3$ PUFA include $\alpha$-linolenic acid (LNA; 18:3n-3 PUFA), DHA (22: 6n-3 PUFA) and EPA (20:5n-3 PUFA). Unlike EPA, dietary DHA has been shown to concentrate specifically in phospholipids of brain cells, while LNA is virtually absent from nervous tissue $^{(1-3)}$. Importantly, it is well known that high dietary consumption of DHA quickly translates into higher brain concentration in mammals ${ }^{(4-8)}$. Besides their well-accepted lowering effect on plasma TAG and on the risk of $\mathrm{CHD}^{(9)}$, preclinical, observational and intervention studies suggest that n-3 PUFA may exert beneficial effects on cognitive/memory performances $^{(10,11)}$ and, possibly, mood disorders ${ }^{(12)}$, as well as Parkinson's ${ }^{(8)}$ and Alzheimer's diseases ${ }^{(13-16)}$. The potential bioactivities of DHA in the brain are numerous and complex. For example, DHA has been reported to regulate membrane properties such as fluidity, elasticity and rigidity ${ }^{(17,18)}$ and to influence cell signalling ${ }^{(19,20)}$, gene expression ${ }^{(21,22)}$, neuroinflammation $^{(23-25)}$ and synaptic health ${ }^{(7,19)}$. More specifically, DHA is thought to play an important role in synapse health by modulating key dendrite proteins, like drebrin and syntaxin-3. The association of drebrin with actin filaments is critical for the stability and plasticity of dendritic spines ${ }^{(26)}$, and DHA intake prevents drebrin loss in the Tg2576 mouse model of Alzheimer's disease ${ }^{(19)}$. Syntaxin-3 is present in synaptic membranes ${ }^{(27)}$ and in neuronal growth cones ${ }^{(28)}$. DHA dietary supplementation elevates synaptic levels of syntaxin- $3^{(29)}$, and the action of DHA on promoting neurite outgrowth and membrane expansion has been shown to depend on syntaxin- $3^{(28)}$. In addition, we recently reported direct evidence that chronic consumption of DHA alters the basic electrophysiological properties of entorhinal cortex (EC) neurons ${ }^{(30)}$.

Abbreviations: AA, arachidonic acid; CC, cell capacitance; DS, detergent-soluble; EC, entorhinal cortex; FA, fatty acids; LNA, $\alpha$-linolenic acid; sEPSC, spontaneous excitatory postsynaptic currents; TBS, Tris-buffered saline.

*Corresponding author: Dr F. Calon, fax +1 4186542761 , email frederic.calon@pha.ulaval.ca 
In the diet, LNA is mainly obtained from oily seeds, whereas DHA and EPA are found in fish and seafood ${ }^{(1)}$. In addition, LNA, EPA and DHA are among the most frequently consumed nutritional supplements. Due to its highest concentration in the brain, DHA is likely to be the most neuroactive $n$-3 PUFA. However, it remains unclear to what extent dietary LNA, through its hepatic conversion into EPA and DHA, can ultimately achieve the same physiological effect ${ }^{(2,31-33)}$. Indeed, despite the fact that the enzymatic machinery to elongate LNA into EPA/DHA is present in the mammal liver, the capacity of this process to actually produce DHA from LNA in significant amounts is disputed, particularly in humans $^{(2,33,34)}$. While it has been clearly demonstrated in animal studies that a high intake of LNA increases brain DHA concentrations ${ }^{(2,11,33)}$, most reports comparing DHA $v$. LNA intake indicate that the maximal concentration of DHA in the brain can only be achieved by including preformed DHA in the food ${ }^{(2,11,33)}$. Notwithstanding these observations, the real question whether using LNA can be as efficient as DHA to achieve central nervous system (CNS) health benefit remains open and needs to be addressed because both types of $n$-3 PUFA are widely available in food or supplements. In addition, although the supply of LNA through vegetables appears sustainable, consumption of DHA up to current health recommendations raises legitimate concerns, given the decline in fish stocks worldwide ${ }^{(35)}$.

To determine whether LNA is equally efficient at supplying enough DHA to the CNS for its optimal functioning, we exposed C57/BL6 mice to either an n-3 PUFA-deficient diet, an LNA diet or a DHA diet. Animals were fed with the three diets, from 4 months to 14.5 months of age, and we then recorded the intrinsic and synaptic properties of EC deeplayer neurons using whole-cell patch-clamp on brain slices. For our electrophysiological analysis, we selected EC neurons because the EC-hippocampus loop plays a key role in higher cognition/memory functions ${ }^{(36)}$, which, as mentioned earlier, are thought to depend on $n$-3 PUFA intake. EC neurons are also known to play a determining role in temporal lobe epilepsy ${ }^{(37,38)}$ and are among the first neurons affected in Alzheimer's disease ${ }^{(39-42)}$

\section{Experimental methods}

\section{Animals and diets}

The use of animals was approved by the Laval University (Quebec, Canada) Animal Ethics Committee (approval ID $=07-113$ and 07-061) and all procedures were performed according to the guidelines of the Canadian Council on Animal Care. Animals were exposed to three treatments: (1) a control safflower oil-based diet ( $n$-3 PUFA-deficient diet); (2) a rapeseed:soyabean (4:1)-based diet containing only LNA as a source of $n$-3 PUFA (LNA diet); (3) a fish oil-based diet containing a high amount of DHA with a lower content in EPA (DHA diet). Ingredients and fatty acid analysis of each diet are detailed in Table 1 . The LNA diet contained a higher proportion of C18:1n-9 MUFA than the two others due to the presence of rapeseed oil. This formula is representative of human-relevant
Table 1. Description of $n-3$ PUFA-deficient, $\alpha$-linolenic acid (LNA) and DHA diets

\begin{tabular}{|c|c|c|c|}
\hline & $n$-3 PUFA deficient & LNA & $\mathrm{DHA}$ \\
\hline \multicolumn{4}{|l|}{ General information ( $\%$ w/w) } \\
\hline Protein & $20 \cdot 3$ & $20 \cdot 3$ & $20 \cdot 3$ \\
\hline Carbohydrate & $66 \cdot 0$ & $66 \cdot 0$ & $66 \cdot 0$ \\
\hline Fat & $5 \cdot 0$ & $5 \cdot 0$ & 4.8 \\
\hline Energy $(\mathrm{kJ} / \mathrm{g})$ & 63.6 & 63.6 & 63.6 \\
\hline Cholesterol & 0.6 & 0.6 & 0.6 \\
\hline \multicolumn{4}{|l|}{ Ingredients (\% w/w) } \\
\hline Casein & 20 & 20 & 20 \\
\hline Maize starch & 15 & 15 & 15 \\
\hline Sucrose & 50 & 50 & 50 \\
\hline Cellulose & 5 & 5 & 5 \\
\hline Mineral mix S19101 & 3.5 & 3.5 & 3.5 \\
\hline Vitamin mix V15908 & 1 & 1 & 1 \\
\hline Maize oil & 2.5 & 0 & 0.4 \\
\hline Safflower oil & 2.5 & 0 & 1.7 \\
\hline Soyabean oil & 0 & 1 & 0 \\
\hline Rapeseed oil & 0 & 4 & 0 \\
\hline MEG-3 powder* (14\% DHA) & 0 & 0 & 4.5 \\
\hline \multicolumn{4}{|l|}{ Fatty acid content $(\mu \mathrm{mol} / \mathrm{g}) \dagger$} \\
\hline C18:2n-6 (LA) & $86 \cdot 8$ & 47.9 & 34.7 \\
\hline C18:3n-3 (LNA) & 0.8 & $12 \cdot 1$ & 0.5 \\
\hline $\mathrm{C} 20: 4 n-6(\mathrm{AA})$ & 0.0 & 0.0 & 1.5 \\
\hline $\mathrm{C} 20: 5 n-3$ (EPA) & 0.0 & 0.0 & 3.0 \\
\hline C22: 4n-6 (DTA) & 0.0 & 0.0 & 0.0 \\
\hline $\mathrm{C} 22: 5 n-6$ (DPA) & 0.0 & 0.0 & 0.7 \\
\hline $\mathrm{C} 22: 6 n-3(\mathrm{DHA})$ & 0.0 & 0.0 & 9.5 \\
\hline$n-3$ PUFA & 0.8 & $12 \cdot 1$ & $13 \cdot 0$ \\
\hline$n-6$ PUFA & $86 \cdot 8$ & 47.9 & $37 \cdot 0$ \\
\hline $\mathrm{C} 18: 2 n-6 / \mathrm{C} 18: 3 n-3$ & $106 \cdot 0$ & 4.0 & 69.4 \\
\hline Total PUFA & 87.8 & $60 \cdot 0$ & $50 \cdot 0$ \\
\hline
\end{tabular}

LA, linoleic acid; AA, arachidonic acid; DTA, docosatetranoic acid; DPA, docosapentaenoic acid.

* Ocean Nutrition Inc., Halifax, Canada

$\dagger$ Measured by GC.

dietary sources of LNA, which generally combine MUFA with LNA. The DHA source was a high-DHA formulation (MEG-3) from Ocean Nutrition Inc. (Halifax, Canada) in which DHA and EPA are microencapsulated into gelatin beads for protection against oxidation $^{(43-45)}$. MEG-3 contains a DHA:EPA ratio of at least 3:1, both in a TAG form. In the LNA diet, the daily dose of LNA was approximately $1.5 \mathrm{mmol} / \mathrm{kg}$ per $\mathrm{d}$, whereas the daily dosage of DHA and EPA was 1.14 and $0.36 \mathrm{mmol} / \mathrm{kg}$ per $\mathrm{d}$, respectively, in the DHA diet (assuming that a $25 \mathrm{~g}$ mouse eats $3 \mathrm{~g}$ of food $/ \mathrm{d}$ ). Only purified diets with precisely determined formulae were used in the present study to avoid any batch-to-batch variations. The three diets were isoenergetic and were exactly the same in terms of total fat $(5 \% \mathrm{w} / \mathrm{w})$, total carbohydrates $(66 \% \mathrm{w} / \mathrm{w})$, total protein $(20 \% \mathrm{w} / \mathrm{w})$, total energy ( $4 \mathrm{kcal} / \mathrm{g}$ or $16.3 \mathrm{~kJ} / \mathrm{g}$ ), fibres, vitamins, minerals and antioxidants, and did not contain phyto-oestrogens unlike most laboratory chows (Table 1). A small difference in the total fat content ( $4.8 \%$ in the DHA diet instead of $5 \%$ ) was probably due to small variation in the estimated value $v$. the actual measured values after incorporation of the MEG-3 formulation. The dietary treatment lasted 10 months, starting at 4 months of age and ending at killing of the animal between 14 and 15 months of age (14.5 (SEM 0.5) months). This treatment period was selected to study the effects of diet in adult animals and to avoid interfering in the developmental or maturation process. 


\section{Preparation of tissue samples}

All the experiments were performed in the same animals. The right hemisphere was devoted for electrophysiology studies. The left hemisphere was quickly dissected and the frontal cortex was used for lipid measurements and the parietotemporal cortex was assigned for Western immunoblots. It was not possible to use the same tissue for both biochemical procedures, as fatty acids (FA) are very sensitive to oxidation. However, previous analyses in rodents and non-human primates have shown that the fatty acid profile is similar between the cortical areas and are affected evenly by DHA supplementation $^{(46-49)}$.

\section{Protein extraction}

After adding eight volumes of Tris-buffered saline (TBS) containing Complete ${ }^{\mathrm{TM}}$ protease inhibitors cocktail (Roche, Indianapolis, IN, USA), $10 \mu \mathrm{g} / \mathrm{ml}$ of pepstatin A, $0 \cdot 1 \mathrm{mm-EDTA}$ and phosphatase inhibitors ( $1 \mathrm{~mm}$ each of sodium vanadate and sodium pyrophosphate, $50 \mathrm{~mm}$ sodium fluoride), the frozen samples were sonicated briefly $(3 \times 10 \mathrm{~s})$ and centrifuged at $100000 \mathrm{~g}$ for $20 \mathrm{~min}$ at $4^{\circ} \mathrm{C}$ to generate a TBS-soluble intracellular and extracellular fraction (soluble fraction). The TBS-insoluble pellet was sonicated in five volumes of lysis buffer $\left(150 \mathrm{~mm}-\mathrm{NaCl}, 10 \mathrm{~mm}-\mathrm{NaH}_{2} \mathrm{PO}_{4}, 1 \%\right.$ Triton X-100, 0.5\% SDS and $0.5 \%$ deoxycholate) containing the same protease and phosphatase inhibitor cocktail. The resulting homogenate was centrifuged at $100000 \mathrm{~g}$ for $20 \mathrm{~min}$ at $4^{\circ} \mathrm{C}$ to produce a lysis/detergent-soluble (DS) fraction (membrane fraction).

\section{Western immunoblotting}

For Western immunoblotting, protein concentration was determined using bicinchoninic acid assays (Pierce, Rockford, IL, USA). Equal amounts of protein per sample ( $15 \mu \mathrm{g}$ of total protein per lane) were added to Laemmli's loading buffer, heated to $95^{\circ} \mathrm{C}$ for $5 \mathrm{~min}$ before loading and subjected to SDS-PAGE. Proteins were electroblotted onto polyvinylidene fluoride membranes (Millipore, Billerica, MA, USA) before blocking in 5\% non-fat dry milk and $1 \%$ bovine serum albumin in PBS-Tween 20 for $1 \mathrm{~h}$. Membranes were immunoblotted with appropriate primary and secondary antibodies followed by chemiluminescence reagents (Lumiglo Reserve; KPL, Gaithersburg, MD, USA). Band intensities were quantified using a KODAK Image Station 4000 MM Digital Imaging System (Molecular Imaging Software version 4.0.5f7; Carestream Health, Rochester, NY, USA). The following antibodies were used in this study: anti-actin (ABM, Richmond, Canada), anti-drebrin (MBL International, Woburn, MA, USA), antipostsynaptic density protein 95 (Millipore), anti-synaptophysin (Millipore), anti-syntaxin 3 (Sigma, St Louis, MO, USA).

\section{Lipid extraction and GC}

Lipid profiles were determined in the frontal cortex, as the fatty acid profile is reported to be similar throughout the brain $\operatorname{cortex}^{(46,47)}$. GC experiments were performed as previously described $^{(8,30,50)}$. Approximately $20 \mathrm{mg}$ of frozen frontal cortex from each mouse were used for lipid extraction. Weighed brain tissues were homogenised with butylated hydroxytoluene-methanol (Sigma) and two volumes of $\mathrm{CHCl}_{3}$ (J.T. Baker, Phillipsburg, NJ, USA) and 0.5 volumes of $\mathrm{NaH}_{2} \mathrm{PO}_{4}(0 \cdot 2 \mathrm{M})$-buffer solution were added to the resulting homogenate. After centrifugation at $2500 \mathrm{~g}$, the lower layer $\left(\mathrm{CHCl}_{3}\right.$ fraction) was collected ${ }^{(51)}$. Lipid extracts were transmethylated with $\mathrm{BF}_{3}-$ methanol (Alltech, State College, PA, USA) at $100^{\circ} \mathrm{C}$ for $60 \mathrm{~min}$. After cooling down, $2 \mathrm{ml}$ of water and $2 \mathrm{ml}$ of hexane (J.T. Baker) were added. After homogenisation and centrifugation at $2500 \mathrm{~g}$, the upper layer (hexane fraction) was collected, dried down to about $100 \mu \mathrm{l}$ and transferred to a GC autosampler vial and capped under $\mathrm{N}_{2}$. Fatty acid methyl ester profiles in brain tissue were obtained by capillary GC using a temperature gradient on an HP5890 gas chromatograph (Hewlett Packard, Toronto, Canada) equipped with an HP-88 capillary column $(100 \mathrm{~m} \times 0.25 \mathrm{~mm}$ inner diameter $\times 0.20 \mu \mathrm{m}$ film thickness; Agilent Technologies, Mississauga, ON, Canada) coupled with a flame ionisation detector. Helium was used as a carrier gas (split ratio 1:80). FA were identified according to their retention time, using the following standard mixtures as a basis for comparison: the FAME 37 mix (Supelco, Inc., Bellefonte, PA, USA) and the GLC-411 fatty acid mix (NuChek Prep, Inc., Elysian, MN, USA), as well as the following methylated FA C22 : 5n-6 (Larodan AB, Malmö, Sweden) and C22:5n-3 (Supelco, Inc.). Finally, a mixture of trans-FA containing: C18:2n-6 cis/trans (Supelco, Inc.), a mixture of cis/trans C18:3n-3 (Supelco, Inc.), the following Nucheck FA: C14:1 trans-9, C16:1 trans-9, and finally, the isoforms of C18: 1 (cis-6, trans-6, cis11, trans-11: Nucheck, and cis-12, cis-13; Supelco, Inc.) were also used as a standard mixture.

\section{Slice preparation for electrophysiology recordings}

Brain slices were prepared as previously described ${ }^{(52,53)}$. Briefly, mice were deeply anaesthetised with ketamine (100 mg/kg, intraperitoneally) and xylazine $(10 \mathrm{mg} / \mathrm{kg}$, intraperitoneally), and decapitated. The brain was removed quickly $(<60 \mathrm{~s})$ and placed in an ice-cold solution containing $(\mathrm{mm})$ : 210 sucrose, $3 \cdot 0 \mathrm{KCl}, 1 \cdot 0 \mathrm{CaCl}_{2}, 3 \cdot 0 \mathrm{MgSO}_{4}, 1 \cdot 0 \mathrm{NaH}_{2} \mathrm{PO}_{4}, 26$ $\mathrm{NaHCO}_{3}$ and 10 glucose, and saturated with $95 \% \mathrm{O}_{2}: 5 \% \mathrm{CO}_{2}$. Horizontal slices of $300 \mu \mathrm{m}$ were cut from inferior to superior brain with a vibrating tissue slicer (VT 1000 s; Leica, Wetzlar, Germany) and kept in artificial cerebral spinal fluid containing (mM): $124 \mathrm{NaCl}, 3 \cdot 0 \mathrm{KCl}, 1.5 \mathrm{CaCl}_{2}, 1 \cdot 3 \mathrm{MgCl}_{2}, 1 \cdot 0 \mathrm{NaH}_{2} \mathrm{PO}_{4}, 26$ $\mathrm{NaHCO}_{3}$ and 20 glucose, and saturated with 95\% $\mathrm{O}_{2}: 5 \%$ $\mathrm{CO}_{2}$ at room temperature $\left(21-23^{\circ} \mathrm{C}\right)$. Slices were allowed to recover for at least $1 \mathrm{~h}$ before recording.

\section{Patch-clamp recording}

For recording, a slice was transferred to a submerge-type chamber and continuously exposed to artificial cerebral spinal fluid heated to $30-32^{\circ} \mathrm{C}$, saturated with $95 \% \mathrm{O}_{2}: 5 \%$ $\mathrm{CO}_{2}$ and flowing at a rate of $2.0($ SEM 0.2$) \mathrm{ml} / \mathrm{min}$. The slices were viewed first with a $4 \times$ objective and a deep layer of

\footnotetext{
cortex (46,47) GC experiments were performed as previously
} 


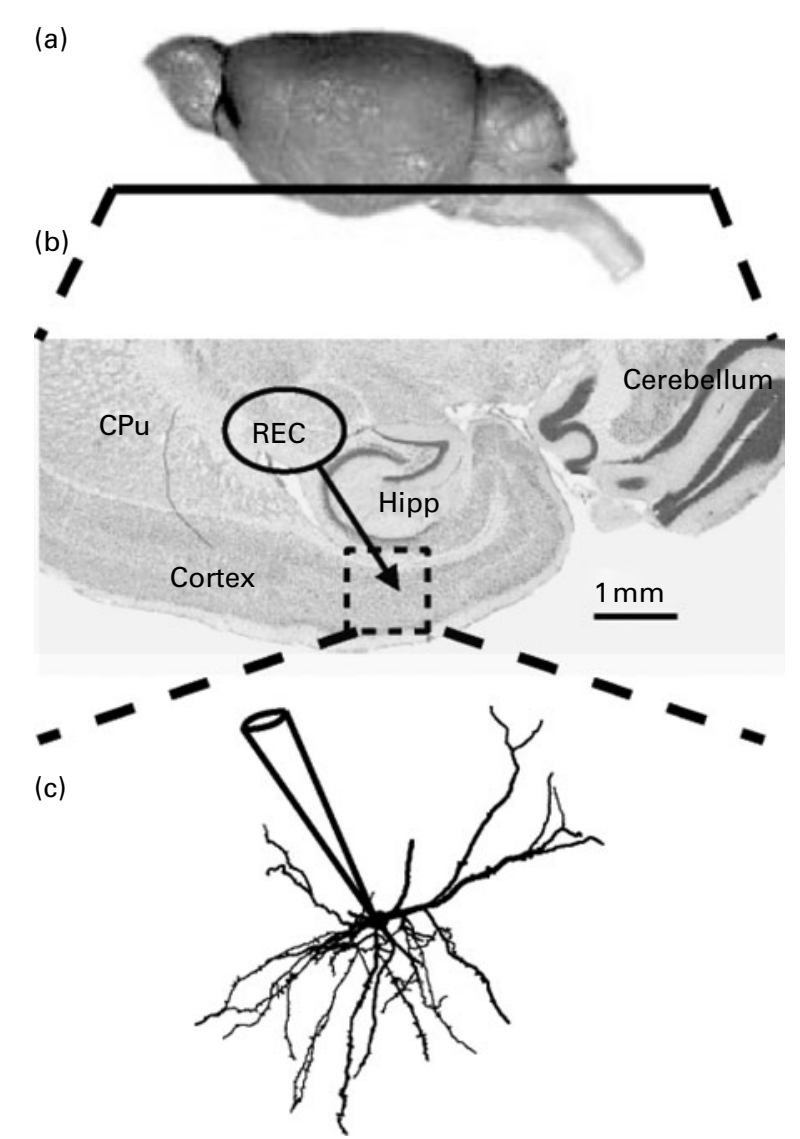

Fig. 1. Electrophysiology methodology. (a) Preparation of slices by horizontal cutting. The black line illustrates the cutting axis. (b) Brain slice stained with haematoxylin. The recorded neurons are those of deep layers of lateral entorhinal cortex. CPu, Caudate putamen (striatum); Hipp, hippocampus; REC, recording electrode. (c) Illustration of the patch-clamp technique. The neuron is labelled with neurobiotin and revealed by immunohistology (nickel3,3'diaminobenzidine tetrahydrochloride (DAB) revelation).

EC was located beside the hippocampus (Fig. 1). In general, two slices can be recorded per hemisphere. Large deeplayer neurons in the EC were then viewed under near-IR illumination with a $40 \times$ water-immersion objective (Fluor, $40 \times$, 0.80W; Nikon, Mississauga, Canada) and a charge-coupled device camera (IR-1000; Dage MTI, Michigan City, IN, USA).

Experiments were conducted at $30-32^{\circ} \mathrm{C}$. Patch pipettes were pulled from thick wall borosilicate glass $(1.5 / 0.84 \mathrm{~mm}$; WPI, Sarasota, FL, USA) on a horizontal puller (P-97; Sutter Instruments, Novato, CA, USA). The pipette solution contained (mM): $100 \mathrm{KMeSO}_{4}, 15 \mathrm{KCl}, 4 \mathrm{ATP}-\mathrm{Mg}, 10$ creatine phosphate, 10 HEPES, 0.5 ethylene glycol tetraacetic acid $(\mathrm{pH} 7 \cdot 2$, adjusted with $\mathrm{KOH}$ ). Electrodes had resistances between 2 and $5 \mathrm{M} \Omega$. Liquid junction potential, estimated to be $5 \mathrm{mV}$, was not corrected. The seal resistance was greater than $2 \mathrm{G} \Omega$. Whole-cell recordings were made at the soma with a Multiclamp 700A amplifier (Molecular Devices, Sunnyvale, CA, USA). The serial resistance, usually between 7 and $20 \mathrm{M} \Omega$, was not compensated. Experiments were conducted using the Axograph 4.9 program (Molecular Devices). Data were digitised at 8 or $16 \mathrm{kHz}$ and were either filtered at $1 \mathrm{kHz}$, depending on the recording protocol.

\section{Data analysis for electrophysiology experiments}

AxoGraph 4.9 (Molecular Devices Corporation, Downingtown, PA, USA) and Origin 7 (OriginLab, Northampton, MA, USA) software programs were used for analysis. Passive properties were studied in current clamp mode, whereas spontaneous excitatory postsynaptic currents (sEPSC) were quantified in voltage-clamp mode with a voltage clamp to $-60 \mathrm{mV}$. The cell conductance (represented by $G_{\mathrm{c}}$ in formula) was estimated from the slope of the graph of hyperpolarised current injection (represented by $I$ in formula) $v$. voltage variation (represented by $V$ in formula). The calculation was derived from the equation $I=G_{\mathrm{c}} \times V$. The injected current duration was $400 \mathrm{~ms}$ and current intensities were 50, 100, 150 and $200 \mathrm{pA}$. Cell capacitance (represented by CC in formula) (i.e. for a first-order resistance-capacitance circuit) was estimated from the linear slope of the plot of $I \times T=\mathrm{CC} \times V$, where $T$ is the time constant of voltage variation, measured by fitting a single exponential function for a voltage decay over time, $V=V_{\infty}\left(1-e^{-T /(R \times C C)}\right)$, where $R$ is the input resistance (i.e. $G_{\mathrm{c}}^{-1}$ ) and $V_{\infty}$ is the asymptote, so that $T=R C$ (i.e. $V=0.632 V_{\infty}$ ), using a graphical method. CC corresponds to the linear slope of the graph displaying the relationship between $I \times T v$. $V$. Passive properties were studied in current-clamp mode because this recording configuration is more accurate than voltage-clamp mode ${ }^{(54)}$. The membrane conductance (represented by $G_{\mathrm{m}}$ in formula; conductance for $1 \mathrm{~F}$ ) was calculated from the resistance and capacitance of the neuron using the formula $G_{\mathrm{m}}=G_{\mathrm{c}} /$ CC. The sEPSC were automatically detected using the event detection package of AxoGraph. This package uses a pre-established template (amplitude: $5 \mathrm{pA}$, rise-time: $0.6 \mathrm{~ms}$, decay time: $3.5 \mathrm{~ms}$, baseline: $1.5 \mathrm{~ms}$, length: $4 \mathrm{~ms}$ ) to detect synaptic events. Events with amplitude $<4 \mathrm{pA}$ were rejected.

\section{Statistical analysis}

Values were expressed as means with their standard errors. Statistical comparisons were performed using a one-way ANOVA followed by the Tukey-Kramer post hoc test. Correlation analyses were performed using linear regressions. Percentage of spontaneously active neurons was analysed by contingency tables using Pearson's $\chi^{2}$ test.

\section{Results}

\section{Effects of PUFA treatment on brain fatty acid profiles}

Several studies have emphasised the critical influence of dietary fat composition on the accumulation of specific FA in the brain $^{(8,55-57,58)}$. To confirm the efficacy of our dietary treatment, we determined the fatty acid profile in the frontal cortex of each animal. The most relevant results are shown in Fig. 2, whereas the detailed data are given in Table S1 of the supplementary material (available online http://www. journals.cambridge.org/bjn). The dietary treatments did not significantly modulate total SFA (approximately 44\%) and total MUFA (approximately 21\%), but total PUFA were 
(a)

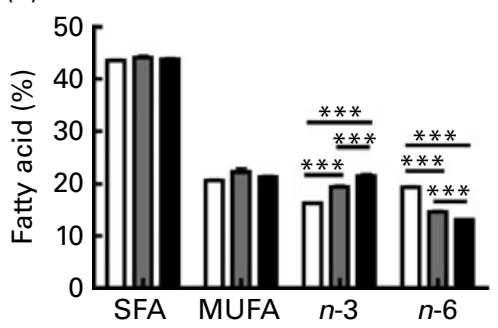

(b)

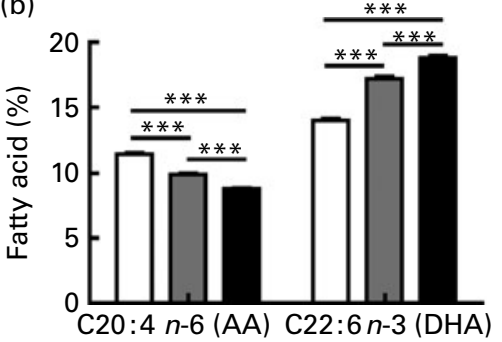

(c)

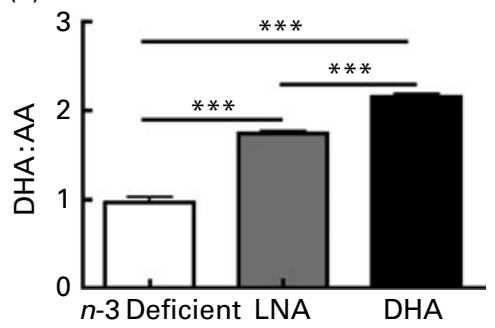

Fig. 2. Effects of $\alpha$-linolenic acid (LNA, $\square$ ) and DHA ( $\square$ ) consumption on the relative percentage of fatty acid in frontal cortex. (a) Dietary DHA increased the amount of total $n$-3 PUFA and decreased total $n-6$ PUFA compared to $n$-3 PUFA-deficient diet ( $\square$ ). The LNA-enriched diet produced an intermediate effect on $n-3$ PUFA and $n-6$ PUFA levels. SFA and MUFA levels were not modified by diets. (b) Chronic DHA intake increased the proportion of DHA and reduced the proportion of arachidonic acid (AA) in the frontal cortex, compared to $n-3$ PUFA-deficient diet. Again, the LNA diet produced an intermediate effect. (c) Consumption of both LNA or DHA increased the DHA:AA ratio, compared to $n$-3 PUFA-deficient diet, but the effect of DHA was significantly more pronounced. Values are means, with their standard errors represented by vertical bars, $n 9$ for the $n-3$ PUFA-deficient group and $n 13$ for LNA and DHA groups. Statistical comparisons were performed using a one-way ANOVA followed by a Tukey-Kramer post hoc test. ${ }^{* * *}$ Mean values were significantly different $(P<0 \cdot 001)$.

slightly lower following LNA intake $(-4$ to $-6 \% ; P<0.05)$. DHA accounted for $85-88 \%$ of cerebral $n-3$ PUFA, while levels of EPA remained very low and LNA was undetectable (Fig. 2 and Table S1, supplementary material for this article can be found at http://www.journals.cambridge.org/bjn). As expected, DHA consumption increased the concentrations of DHA and total $n-3$ PUFA in the brain compared to the other two diets $(32-34 \%$ compared to the $n-3$ PUFA-deficient diet and $9-11 \%$ compared to the LNA diet; $P<0 \cdot 001)$. Conversely, we observed a decrease of arachidonic acid (AA; C20 : 4n-6 PUFA; $-23 \%$ compared to the $n-3$ PUFA-deficient diet and $-11 \%$ compared to the LNA diet; $P<0.001)$ and total $n-6$ PUFA ( $-33 \%$ compared to the $n-3$ PUFA-deficient diet and $-10 \%$ compared to the LNA diet; $P<0.001)$ following consumption of DHA. LNA intake also increased DHA and $n-3$ PUFA $(P<0 \cdot 001,23 \%$ compared to the $n-3$ PUFA-deficient diet $)$ and decreased AA $(P<0.001,-13 \%$ compared to the $n$-3 PUFA-deficient diet $)$ and $n-6$ PUFA $(P<0 \cdot 001,-25 \%$ compared to the $n-3$ PUFA-deficient diet) in the cortex of mice, but to a lesser degree compared to the diet containing preformed DHA from fish oil $(P<0.001 ;$ Fig. 2$)$. The DHA:AA ratio was thus greatly increased following DHA intake (74\%, $P<0.001$ ), whereas the LNA diet had an intermediate effect ( $41 \%$ compared to the $n$-3 PUFA-deficient diet, $P<0.001 v$. the other two groups, Table $\mathrm{S} 1$, supplementary material for this article can be found at http://www.journals.cambridge. org/bjn). In addition, LNA intake increased the concentrations of C18 : $1 n$-9 MUFA $(12 \%, P<0.05)$ and induced a switch from C18:0 to C16:0 $(P<0 \cdot 05)$. Levels of docosapentaenoic acid (C22:5n-6 PUFA) remained low in DHA or LNA-fed animals, compared to control animals $(P<0 \cdot 001)$. In summary, high chronic intake of DHA massively increased brain concentrations of DHA, whereas the DHA-increasing effect of LNA treatment was highly significant, but less striking.

\section{$\alpha$-Linolenic acid did not modulate passive properties of neurons: comparison with DHA}

We next sought to determine how LNA treatment influenced the basic properties of neurons within the mouse EChippocampal circuitry, compared to DHA (Fig. 3(a) and (b)).
A summary of results is presented in Fig. 3, and given in more detail in Table $\mathrm{S} 2$ of the supplementary material (available online at http://www.journals.cambridge.org/bjn). First, we observed that cell conductance, an indicator of the flux of ions crossing the membrane via ion channels, was higher in DHA-enriched EC neurons $(P<0.05$ compared to the $n-3$ PUFA-deficient diet; Fig. 3(c) and (d)). Second, increased CC, an indicator of total membrane area ${ }^{(54,59,60)}$, was recorded in neurons from animals fed DHA compared to the $n$-3 PUFA-deficient diet $(P<0 \cdot 05$, Fig. 3(e) and (f)) A strong correlation was found between CC and cell conductance $\left(P<0 \cdot 01, r^{2} 0 \cdot 258, n 37\right)$. Third, cellular time constant and membrane conductance (conductance per capacitance unit) were not altered by dietary PUFA. Finally, DHA induced a hyperpolarisation of EC neurons compared to the $n-3$ PUFA-deficient diet $(P<0 \cdot 05$; Fig. $3(\mathrm{~g}))$, whereas animals fed LNA had an intermediate resting potential not significantly different from the $n-3$ PUFA-deficient diet or DHA groups. Consequently, we found an increase of spontaneously active neurons after the $n$-3 PUFA-deficient diet (45\%), compared to LNA- (18\%) and DHA-rich (9\%) diets (Fig. 3(h)). Overall, LNA treatment had little effect on passive properties of EC neurons, suggesting that changes in capacitance, conductance and resting potential occurred only after the brain concentrations of DHA reached a threshold. LNA consumption was nonetheless sufficient to diminish the spontaneous activity of EC neurons.

\section{DHA, but not $\alpha$-linolenic acid, increased spontaneous excitatory post-synaptic currents frequency}

To probe whether LNA consumption changes the network integration of EC neurons, we recorded the sEPSC, an indicator of excitatory synaptic connectivity. We found that neurons from DHA-fed animals detected more sEPSC than those from LNA or $n-3$ PUFA-deficient-fed animals $(P<0 \cdot 05$, Fig. 4(a) and (b) and Table S2, supplementary material for this article can be found at http://www.journals.cambridge. org/bin). However, the increase in sEPSC became undetectable when the frequency of sEPSC was normalised to CC (Fig. 4(c)), strongly suggesting that the rise in sEPSC was a consequence of increased membrane surface area of the neur- 
(a)

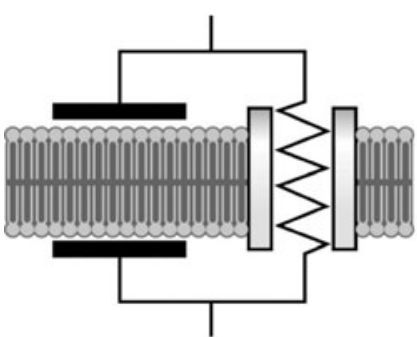

(c)

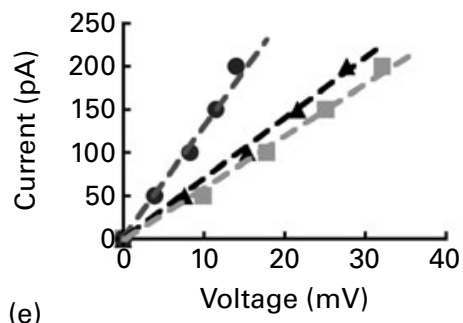

(e)

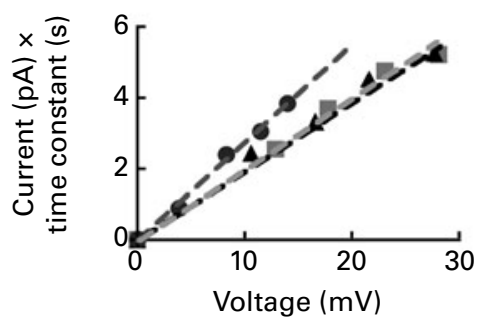

(g)

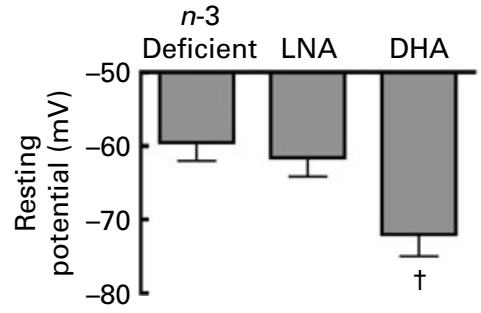

(b)

Hyperpolarising current injection

(d)
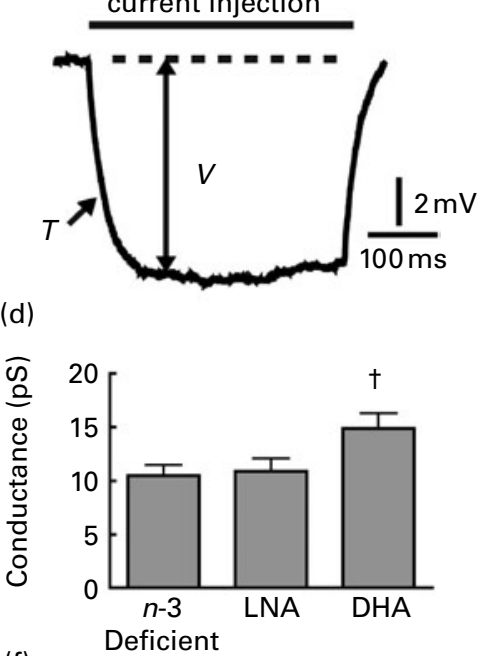

(f)

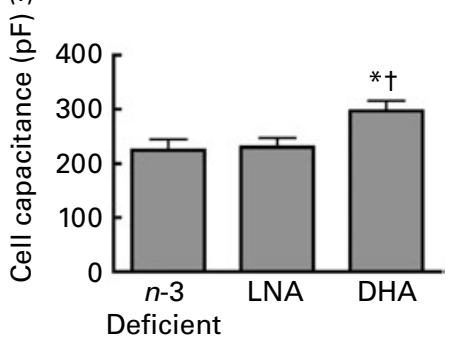

(h)

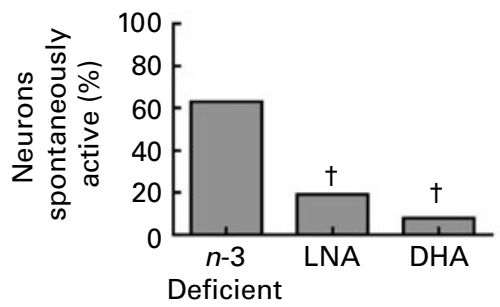

Fig. 3. Chronic DHA intake modulated the passive properties of entorhinal cortex (EC) deep layer neurons. (a) Electrical representation of a cell membrane. (b) To quantify the passive properties, different intensities of hyperpolarising current were injected into the neuron in current clamp and the voltage variation $(V)$ and time constant $(T)$ were measured after each injection. (c) Relationship between the hyperpolarising current injections and resulting $V s$ of EC neurons of mice fed with $n$-3 PUFA-deficient $(\square), \alpha$-linolenic acid (LNA, $\mathbf{A})$ or DHA $(\bullet)$ diet. The slope corresponds to the cell conductance of the neuron. (d) In contrast to DHA, LNA intake did not increase the cell conductance of EC neurons, compared to $n-3$ PUFA-deficient group. (e) Relationship between the injected current $\times T$ and the $V$ recorded in EC neurons of mice fed with the three different diets. The slope of each linear curve corresponds to cell capacitance. (f) In contrast to DHA, LNA intake did not increase the cell capacitance of EC neurons. (g) The membrane potential of neurons was hyperpolarised in mice fed the DHA diet, compared to $n-3$ PUFA-deficient diet. (h) Dietary intake of LNA or DHA decreased the number of spontaneously active EC neurons. Values are means, with their standard errors represented by vertical bars. Statistical comparisons were performed using a one-way ANOVA followed by a Tukey-Kramer post hoc test and a Pearson's $\chi^{2}$ test (\% of neurons spontaneously active). The numbers of recorded neurons were 12,13 and 12 for $n$-3 PUFA-deficient, LNA and DHA groups, respectively. ${ }^{*}$ Mean values were significantly different from those of LNA diet group $(P<0.05)$. † Mean values were significantly different from those of $n-3$ PUFA-deficient group $(P<0.05)$.

ons. The positive correlation between sEPSC and CC supports this hypothesis $\left(P<0 \cdot 05, r^{2} 0 \cdot 2031, n\right.$ 26). The amplitudes of sEPSC remained unchanged between the groups (Fig. $4(d)$ ), suggesting that a similar intensity of electrical or ion transfer was channelled through each synapse.

\section{Implication of syntaxin-3 and drebrin in the regulation of cell capacitance by DHA diet: threshold effect}

The membrane expansion induced by DHA, as indicated by the increase in CC, is consistent with morphological assess- ments of cultured neurons exposed to $\mathrm{DHA}^{(61-63)}$. Two major molecular mechanisms particularly suspected to mediate this effect of DHA involve the synaptic proteins drebrin and syntaxin-3. The role of drebrin in the regulation of postsynaptic cytoarchitecture has been compellingly documented $^{(64,65)}$ and DHA has been shown to prevent the translocation of drebrin from the membrane to the cytosol in Tg2576 mice ${ }^{(19)}$, a model of Alzheimer's disease with neuronal atrophy $^{(66)}$. On the other hand, the binding to syntaxin-3 of long-chain PUFA such as DHA has been identified as a critical step in neurite outgrowth ${ }^{(28)}$. To verify whether drebrin and 
(a)

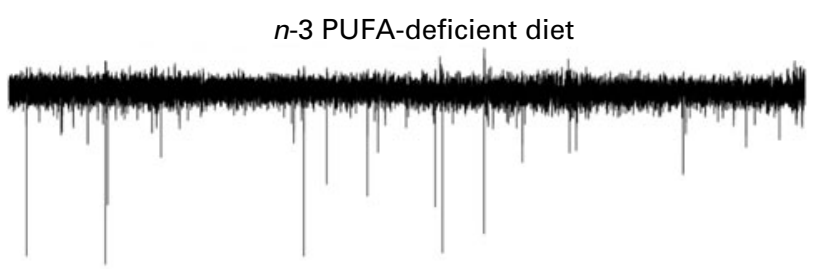

LNA diet
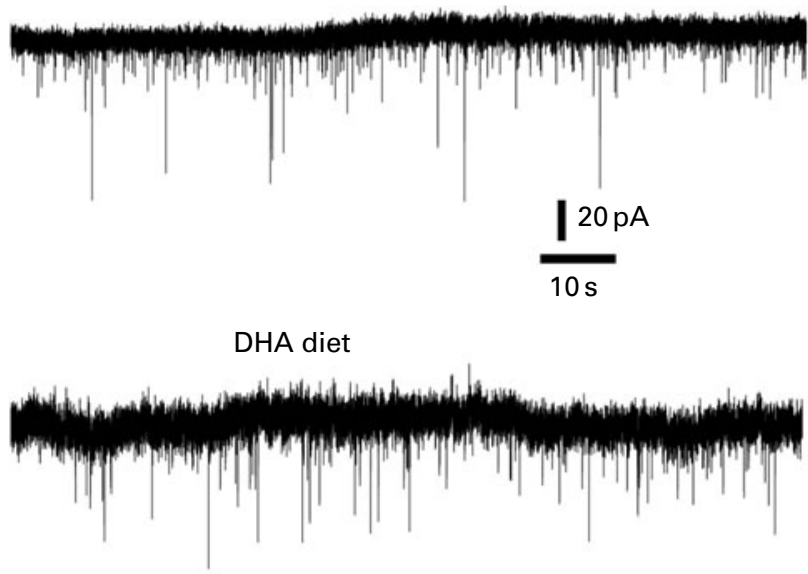

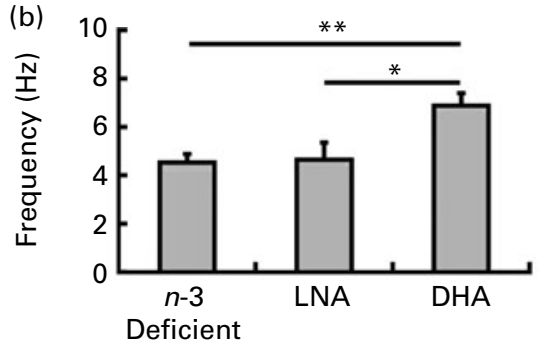

(c)

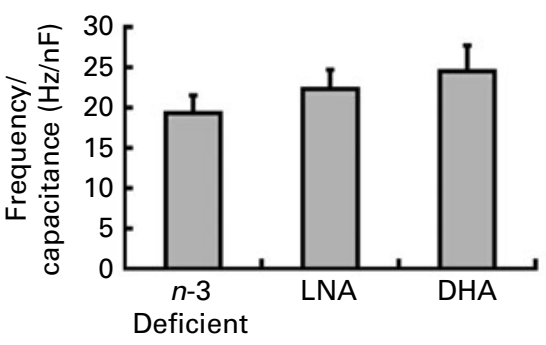

(d)

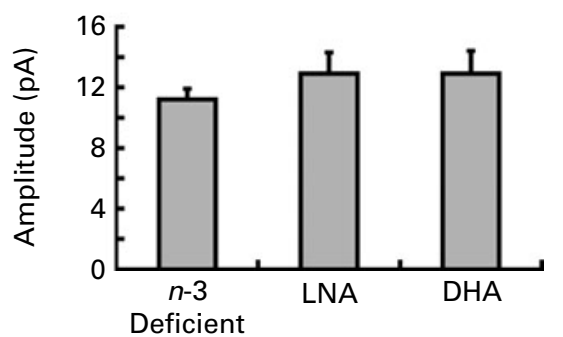

Fig. 4. $\alpha$-Linolenic acid (LNA) consumption did not reproduce the increase in frequency of spontaneous excitatory postsynaptic current (sEPSC) observed after a DHA-enriched diet. (a) Examples of intracellular recordings of sEPSC (voltage clamped at $-60 \mathrm{mV}$ ). (b) Treatment with DHA increased the frequency of sEPSC (c) No difference was found for sEPSC frequency normalised to cell capacitance, an indicator of total membrane surface. (d) sEPSC amplitude was not affected by dietary treatments. Values are means, with their standard errors represented by vertical bars. Statistical comparisons were performed using a one-way ANOVA followed by a Tukey-Kramer post hoc test. The numbers of recorded neurons were 11,7 and 8 for $n$-3 PUFA deficient, LNA and DHA groups, respectively. Mean values were significantly different: ${ }^{*} P<0.05,{ }^{* *} P<0.01$

syntaxin-3 were implicated here, their concentrations were quantified in cytosolic (TBS/soluble fraction) and membrane homogenate preparations (DS fractions) from the parietotemporal cortex of mice. First, we observed that CC correlated positively with syntaxin-3 levels (Fig. 5(a)) and with the translocation ratio (membrane/cytosol: DS/TBS fractions) of drebrin (Fig. 5(b)). In addition, we noted a positive relationship between syntaxin-3 concentrations and the drebrin translocation ratio (Fig. 5(c)), suggesting a link between these two molecular pathways. Second, we found that chronic intake of preformed DHA increased syntaxin-3 levels $(P<0.05$; Fig. 5(d)) and reduced the DS:TBS drebrin ratio $(P<0.05$; Fig. 5(e)), compared to both diets without DHA (LNA and $n-3$ PUFA-deficient diets). Values of syntaxin-3 were $0 \cdot 81$ (SEM $0 \cdot 06 ; n$ 7) for no DHA group and $1 \cdot 19$ (SEM $0 \cdot 14 ; n$ ) for DHA group, whereas they were 0.86 (SEM $0.07 ; n$ 6) and $1 \cdot 20$ (SEM $0 \cdot 12 ; n$ 6) for DS:TBS ratio of drebrin, respectively (Fig. 5(d) and (e)). Thirdly, we found that changes in CC, syntaxin-3 and DS:TBS drebrin ratio were observed only when the DHA:AA ratio reached a certain level (Fig. 5(f)-(h)). Indeed, we noted that a DHA:AA ratio threshold value of $\geq 2: 1$ was necessary to induce an increase in CC (Fig. 5(g)), in syntaxin-3 (Fig. 5(h)) and the translocation ratio of drebrin from the membrane to the cytosol (Fig. 5(i)), as obtained only after high DHA consumption. Altogether, these corroborating observations suggest a strong association between syntaxin-3 concentrations, drebrin translocation and the DHA-induced membrane expansion, and are in accordance with a threshold effect of DHA. Furthermore, the intermediate effect of LNA diet (between $n$-3 PUFA-deficient and DHA diets) was not sufficient to alter any basic parameters of EC cells, except the number of spontaneously active neurons.

\section{Discussion}

The data generated in the present study are consistent with the following conclusions. First, chronic LNA consumption increased DHA and reduced AA concentrations in the brain cortex, but to a lesser extent than treatment with an equimolar daily dose of preformed DHA. Second, LNA treatment did not replicate the DHA-induced increases in CC and SEPSC frequencies. Third, the effect of DHA on CC was strongly associated with increased concentrations of syntaxin-3 and drebrin translocation towards the membrane fractions. The effect of 

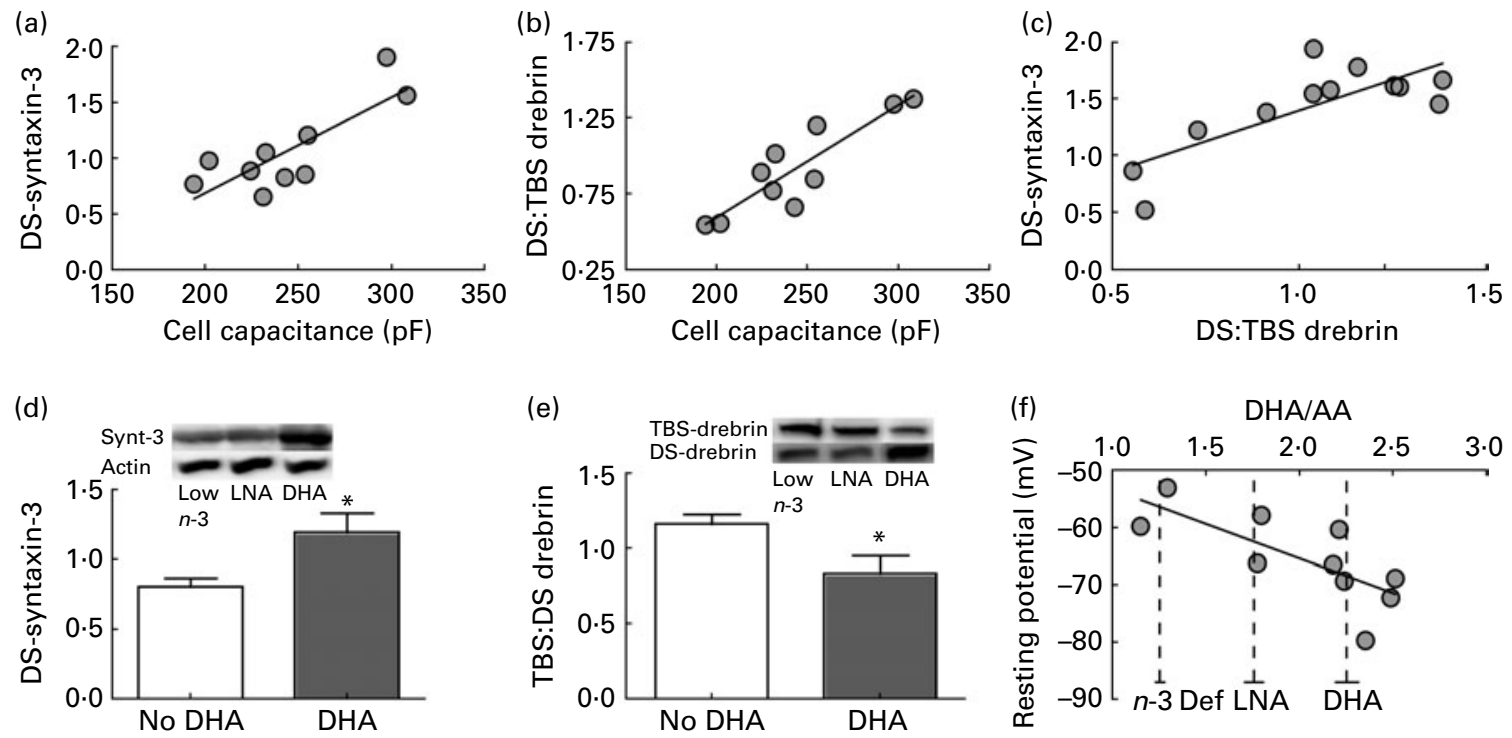

(g)

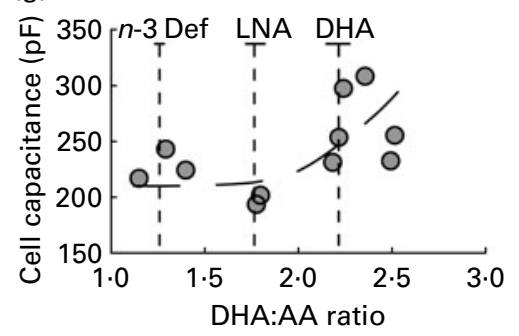

(h)

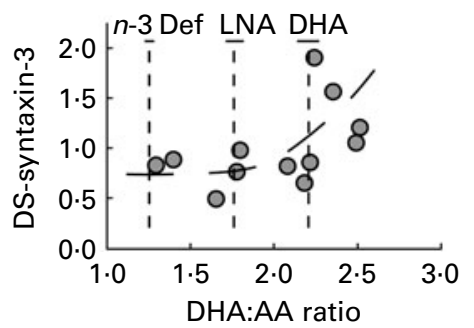

(i)

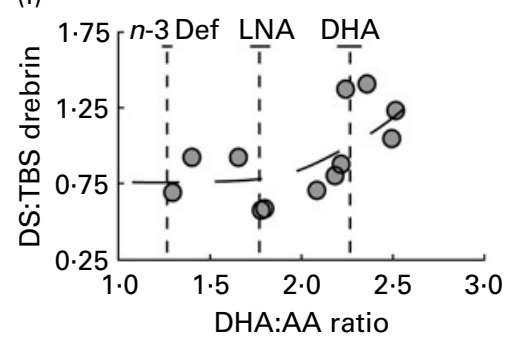

Fig. 5. Association between drebrin and syntaxin-3 and cell capacitance. Cell capacitance positively correlated with (a) syntaxin-3 levels $\left(r^{2} 0.63, P<0.01\right)$ and (b) detergent-soluble (DS):Tris-buffered saline (TBS) drebrin translocation ratio $\left(r^{2} 0.62, P<0.01\right)$, whereas a positive relationship was observed between (c) syntaxin-3 and the drebrin translocation ratio $\left(r^{2} 0.57, P<0.01\right)$. Dietary intake of DHA led to (d) an increase in syntaxin-3 and $(e)$ a decrease in the drebrin translocation ratio, compared to $n$-3 PUFA-deprived control animals ( $d$ and e). While the DHA:arachidonic acid (AA) ratio was linearly associated with (f) resting potential $\left(r^{2} 0.41, P<0.05\right)$, (g) cell capacitance, (h) DS syntaxin-3 and (i) the DS:TBS drebrin translocation ratio were altered after the DHA:AA ratio reached approximately 2:1 in the brain. The dotted line on each graph represents the average DHA:AA ratio following each diet, whereas the horizontal bar at the end represents the standard error mean. Statistical comparisons were performed using unpaired $t$ test $(\mathrm{d}$ and $\mathrm{e})$ or linear regression $(\mathrm{a}, \mathrm{b}, \mathrm{c}, \mathrm{f}) .{ }^{*}$ Mean values were significantly different from those of animal receiving no DHA $(P<0 \cdot 05) . n-3$ Def, $n-3$ PUFA deficient.

brain DHA on CC, syntaxin-3 and drebrin appears to occur above a DHA:AA ratio threshold, reachable only with dietary intake of preformed DHA diet and not with intake of LNA. Finally, treatment with DHA hyperpolarised the resting potential of EC neurons and decreased the number of spontaneously active neurons, whereas LNA consumption led to an intermediate effect on these electrophysiological parameters.

\section{$D H A$ readily accumulates in the brain: partial conversion of $\alpha$-linolenic acid into DHA}

As expected, dietary consumption of preformed DHA was readily translated in the brain fatty acid profiles. Indeed, the intake of DHA during 10 months led to increased DHA concentrations in the frontal cortex, compared to animals fed the control, $n$-3 PUFA-deficient diet. Such observation is consistent with numerous previous reports ${ }^{(1,7,8,30,47)}$ and it may be explained in part by the fact that DHA readily crosses the blood-brain barrier in vivo ${ }^{(67)}$, where it stably incorporates into cerebral phospholipids ${ }^{(3,68)}$.
The effect of LNA on brain $n$-3 PUFA concentration is less straightforward than preformed DHA. LNA is a metabolic precursor of DHA and mammals have the enzymatic machinery to transform LNA into $\mathrm{DHA}^{(2,33)}$. The important relationship between dietary LNA and brain DHA has been known for decades ${ }^{(69,70)}$, and has been confirmed in many animal studies $^{(1,6-8,71,72)}$. However, studies have also shown that this process may be limited in mammals, and almost negligible in humans exposed to usual diets ${ }^{(2,33)}$. Here, an equimolar intake of LNA led to a significant increase in brain DHA, which did not match the effect of the high-DHA diet, in agreement with previous studies in rodents ${ }^{(2,73)}$. Thus, our results are consistent with the contention that supplementation in preformed DHA is manifold more efficient than an equivalent daily dose of LNA to increase the concentration of DHA in the brain.

Nevertheless, we observed that both LNA and DHA reduced n-6 docosapentaenoic acid concentrations, a result consistent with previous analyses in animals ${ }^{(11,73)}$. Indeed, a brain $n-6$ docosapentaenoic acid elevation indicates a state of DHA deficiency in rodents, probably reflecting desperate attempts by the brain to generate twenty-two carbon PUFA in place 
of the missing $\mathrm{DHA}^{(56,74)}$. This observation is important because it shows that LNA intake alone was efficient to fully reverse the state of DHA deprivation found in animals fed a $n$-3-PUFA-depleted diet. In other words, based on such data, one might expect the LNA-enriched diet to be sufficient to avoid the disruption of PUFA-related brain activities.

\section{$\alpha$-Linolenic acid diet does not achieve the effect of DHA on cell capacitance and the frequency of spontaneous excitatory postsynaptic currents}

Despite the fact that the LNA-enriched diet increased DHA concentration and decreased the level of AA compared to the $n-3$ PUFA-deficient diet, it was apparently not sufficient to induce changes in CC and synaptic activity similar to dietary supplementation of preformed DHA. Thus, the present data suggest that LNA, the essential precursor of $n-3$ PUFA, can be partly converted to DHA, but in insufficient quantity to modulate the CC of EC neurons. An apparent threshold relationship between CC and DHA:AA ratio, reached exclusively with DHA intake, strongly supports this view. In addition, DHA intake modulated the brain concentrations of other bioactive PUFA, such as 22: 5n-3 PUFA, compared to the LNA-based diet (see Table S2 of the supplementary material available online at http://www. journals.cambridge.org/bjn), which could also underlie the present electrophysiological observations. For example, $22: 5 n-3$ ( $n-3$ docosapentaenoic acid) is a known precursor of cyclo-oxygenase 2-dependent electrophilic oxo-derivatives molecules, which are potent anti-inflammatory mediators ${ }^{(75)}$.

Since the capacitance of a given membrane surface area is a constant value for all cells ${ }^{(59)}$, the estimation of decay time constant of voltage variation to calculate CC directly reflects the total membrane surface ${ }^{(54,59)}$. Accordingly, the electrophysiological quantification of CC was previously used to measure membrane atrophy during the neurodegenerative process in an Alzheimer mouse model ${ }^{(30)}$ and to measure the membrane expansion of interneurons during development of Caenorhabditis elegans ${ }^{(60)}$. Such an enlarging effect of DHA on EC cells thus suggests that DHA intake promoted the network integration of neurons in vivo ${ }^{(62)}$. Supporting this idea, we found consequently a higher synaptic detection by DHA-enriched EC neurons. In accordance with our study, DHA promotes neurite outgrowth and synaptic formation in vitro and in vivo ${ }^{(61,62,76)}$, whereas a higher dentritic spine density was reported in hippocampal neurons of gerbil following DHA and uridine-5-monophosphate supplementation ${ }^{(77)}$. The role of DHA in promoting/stabilising excitatory synapses might involve inhibition of glutamate transporters located on astrocytes ${ }^{(78-80)}$, leading to a higher concentration of glutamate in the synapse cleft and prolongation of post- and presynaptic receptor activation ${ }^{(80,81)}$. Such a mechanism could explain the increase of functional synapses observed in the present study after exposure to dietary DHA.

Our correlative analysis suggests that the CC started to rise when the DHA:AA threshold ratio was approximately $2: 1$. This observation suggests that it is the concurrent opposite variation of both PUFA, which underlies their effects on drebrin, syntaxin-3 and CC, although our data do not provide a clear mechanistic explanation. Quite interestingly, this DHA:AA threshold ratio coincided with the increase of syntaxin-3 levels and the translocation of drebrin from the cytosol to the membrane compartment, suggesting an involvement of these two proteins in regulating the size of the neuronal membrane surface. In agreement with our data, DHA administration was shown to increase the level of syntaxin-3 in the brain of rodents ${ }^{(76,82)}$, whereas the binding of DHA to syntaxin-3 induced its conformational change and complexing to SNAP-25 (synaptosomal-associated protein $25 \mathrm{kDa}$ ), thereby promoting membrane expansion ${ }^{(28)}$. Thus, up-regulation of syntaxin-3 is a plausible mechanism for the DHA-induced membrane expansion. Drebrin is another postsynaptic protein involved in dendritic spines scaffold ${ }^{(19,64,65)}$ and required for optimal cognitive function ${ }^{(83)}$. A loss of drebrin by antisense transfection reduced neurite outgrowth in neuroblastoma B104 cells ${ }^{(84)}$ and DHA treatment prevents the loss of drebrin in the Tg2576 model of Alzheimer's disease ${ }^{(19)}$, a mouse line displaying neuronal atrophy ${ }^{(66)}$. Overall, our data suggest that there is a threshold DHA:AA ratio in the brain leading to membrane expansion, in which the postsynaptic proteins, drebrin and syntaxin-3, play a role.

Given the crucial role of EC in cognition and memory, these differential effects of DHA and LNA on the basic electrophysiological properties of EC neurons could be instrumental to their action on CNS function. We have recently proposed that the enhancing effect of DHA on CC may reflect increased membrane surface area of neurons and improved capacity to communicate with other neurons and to collect/process a greater flow of brain information ${ }^{(30)}$. The increased number of sEPSC detected in DHA-enriched neurons supports the idea of an enhanced integration into the neuronal network ${ }^{(30)}$. Thus, these cellular effects of DHA may be directly related to some of the reported effects of DHA on memory/cognition function in animals ${ }^{(11,19,30,85,86)}$ and in humans ${ }^{(16,87)}$. Accordingly, the membrane surface expansion induced by DHA may be therapeutic in neurodegenerative diseases involving neuronal atrophy, such as Alzheimer's disease ${ }^{(30)}$, where the beneficial prophylactic effects of DHA have been reported in animal models ${ }^{(8,13,19,86)}$ and suspected in human subjects ${ }^{(15,88)}$. The present data demonstrate the incapacity of dietary LNA to reproduce the effects of DHA on EC neuron physiology, and thus suggest that LNA may also be unable to exert the same beneficial effect on cognition as DHA, at least when consumed in an equimolar dose. Such contention is supported by a recent study showing that dietary LNA, when compared headto-head with preformed DHA, does not achieve the same protective effect on sensorimotor gating, assessed by prepulse inhibition in $\mathrm{C} 57 \mathrm{Bl} 6$ mice $^{(89)}$. However, previous studies have shown that small increases in brain DHA produced by dietary LNA are enough to modify neurophysiological parameters such as synapse plasticity ${ }^{(90)}$, whereas the sequential administration of three doses of LNA increases neurogenesis and expression of key proteins involved in synaptic function (synaptophysin-1, vesicle-associated membrane protein 2 (VAMP-2), synaptosomal-associated protein $25 \mathrm{kDa}$ (SNAP-25) and vesicular glutamate transporters 1 and 2 (VGLUT1 and VGLUT2) $)^{(91)}$. These two studies clearly show that dietary 
LNA can influence cerebral function, possibly through conversion into DHA or other $n-3$ PUFA.

\section{Regulation of neuronal excitability by n-3 PUFA: network stability and anticonvulsive effect}

LNA treatment did not fully reproduce another critical effect of DHA: the hyperpolarisation of the resting potential of EC neurons. Indeed, exposure to DHA during 10 months led to decrease of resting potential from -60 to $-70 \mathrm{mV}$, whereas the effect of LNA was limited to a few $\mathrm{mV}$. Our correlative analysis established a close link between the DHA:AA ratio and resting potential, suggesting that the limited capacity of LNA to modulate cerebral content of PUFA, compared to the DHA diet was again the most probable explanation. Since membrane hyperpolarisation is a basic mechanism of many anti-epileptic drugs, our results argue in favour of a potential therapeutic role of DHA, and not LNA, in the prevention of epileptic seizures. Anticonvulsant effects of DHA have been suggested by several authors ${ }^{(92-95)}$ and a decrease in frequency and intensity of seizures following DHA intake have been reported in epileptic patients ${ }^{(96)}$. In summary, our data suggest that the change of ionic balance induced by dietary DHA can produce a superior anticonvulsant effect than LNA intake. However, it must be noted that very limited LNA-induced hyperpolarisation was apparently sufficient to reduce significantly the number of spontaneously active neurons. This surprising observation indicates that the consumption of LNA may nevertheless lead to enough brain DHA to reduce the production of non-specific activity of EC neurons within the network.

\section{Nutritional implications}

The study of the intrinsic properties provides basal information about neurons. They describe synaptic integration, the membrane surface, ion flux crossing the membrane and the intracellular ionic balance of each neuron. Thus, the present finding that equimolar dietary LNA cannot achieve the effects of DHA on intrinsic properties of EC neurons has important nutritional implications. Although people are becoming more educated concerning the potential benefit of $n$-3 PUFA, having to choose between LNA and EPA/DHA remains an important practical dilemma. Indeed, LNA and EPA/DHA are found in different foods and different nutritional supplements. Hence, consumption of LNA without an adequate supply of EPA/DHA may lead to sub-optimal brain DHA levels to change the intrinsic properties of neurons. Currently, the market is overwhelmed with products claiming to contain health-promoting $n-3$ PUFA, but containing only LNA without EPA or DHA. The present study provides one of the first direct pieces of evidence that the effect of a chronic dietary intake of preformed DHA on neuronal function cannot be replicated with LNA, despite comparable daily dosage. However, as human and rodent diets typically contain higher amounts of LNA and lower amounts of DHA than the animal diets used in the present study, it is possible that consumption of very high level of LNA could achieve the effect of preformed DHA by its conversion to DHA or other $n$-3 PUFA in the liver ${ }^{(2,31-33)}$. Nevertheless, our results suggest that the beneficial effect of DHA on memory and cognition may be achieved more efficiently with dietary consumption of preformed DHA rather than with LNA.

\section{Conclusion}

We have shown that dietary LNA did not replicate the striking effect of DHA on EC neuronal function, such as changes in CC, resting potential and synaptic activity. Nevertheless, LNA supplementation significantly increased brain DHA levels and decreased AA levels, but not to the extent of direct intake of preformed DHA. The inability of LNA treatment to modulate passive properties of EC neurons may result from its inefficiency to raise the DHA:AA ratio above a threshold necessary to induce an increase of syntaxin- 3 and the translocation of drebrin to the membrane compartment, as these two proteins have been involved in the regulation of membrane/ cellular/neurite expansion. Since the basic properties of EC neurons modulated here by DHA are likely to be essential to higher brain function, our data support the preferential use of preformed DHA rather than LNA in CNS diseases.

\section{Acknowledgements}

This work was supported by grants from the Canadian Institutes of Health Research (FC - MOP74443), the Alzheimer Society Canada (FC - ASC 0516) and the Canada Foundation for Innovation (10307). D. A. held a studentship from the Canadian Institutes of Health Research and the Fonds d'Enseignement et de Recherche of the Faculty of Pharmacy of Laval University. C. J. is supported by the Alzheimer Society Canada, the Fonds de la recherche en santé du Québec and the Fonds d'Enseignement et de Recherche of the Faculty of Pharmacy of Laval University. The work of F. C. was supported by a New Investigator Award from the Clinical Research Initiative and the Canadian Institutes of Health Research Institute of Aging (CAN-76833). Experimental design was made by F. C. and D. A. All of the electrophysiological and molecular experiments were performed by D. A. D. A. and C. J. performed the FA experiments. The management of the animal colony was under the supervision of C. J. The figures and analysis of the results were made by D. A., whereas the manuscript was written by D. A. and F. C. The authors declare that they have no actual or potential conflict of interest. The use of animals was approved by the Laval University Animal Ethics Committee in accordance with the standards of the Canadian Council on Animal Care.

\section{References}

1. Salem N Jr (1989) Omega-3 fatty acids: molecular and biochemical aspects. In New Protective Roles for Selected Nutrients, pp. 109-228 [GA Spiller and J Scala, editors]. New York, NY: Alan R. Liss.

2. Brenna JT, Salem N Jr, Sinclair AJ, et al. (2009) Alpha-linolenic acid supplementation and conversion to $n-3$ long-chain 
polyunsaturated fatty acids in humans. Prostaglandins Leukot Essent Fatty Acids 80, 85-91.

3. Umhau JC, Zhou W, Carson RE, et al. (2009) Imaging incorporation of circulating docosahexaenoic acid into the human brain using positron emission tomography. J Lipid Res $\mathbf{5 0}$, $1259-1268$

4. Farquharson J, Cockburn F, Patrick WA, et al. (1992) Infant cerebral cortex phospholipid fatty-acid composition and diet. Lancet 340, 810-813.

5. Makrides M, Neumann MA, Byard RW, et al. (1994) Fatty acid composition of brain, retina, and erythrocytes in breast- and formula-fed infants. Am J Clin Nutr 60, 189-194.

6. Moriguchi T, Greiner RS \& Salem N Jr (2000) Behavioral deficits associated with dietary induction of decreased brain docosahexaenoic acid concentration. I Neurochem 75, 2563-2573.

7. Calon F, Lim GP, Morihara T, et al. (2005) Dietary $n-3$ polyunsaturated fatty acid depletion activates caspases and decreases NMDA receptors in the brain of a transgenic mouse model of Alzheimer's disease. Eur J Neurosci 22, 617-626.

8. Bousquet M, Saint-Pierre M, Julien C, et al. (2008) Beneficial effects of dietary omega-3 polyunsaturated fatty acid on toxin-induced neuronal degeneration in an animal model of Parkinson's disease. FASEB J 22, 1213-1225.

9. Harris WS (2008) The omega-3 index as a risk factor for coronary heart disease. Am J Clin Nutr 87, 1997S-2002S.

10. Carrie I, Smirnova M, Clement M, et al. (2002) Docosahexaenoic acid-rich phospholipid supplementation: effect on behavior, learning ability, and retinal function in control and $n-3$ polyunsaturated fatty acid deficient old mice. Nutr Neurosci 5, 43-52.

11. Fedorova I, Hussein N, Baumann MH, et al. (2009) An $n-3$ fatty acid deficiency impairs rat spatial learning in the Barnes maze. Behav Neurosci 123, 196-205.

12. Kidd PM (2007) Omega-3 DHA and EPA for cognition, behavior, and mood: clinical findings and structural-functional synergies with cell membrane phospholipids. Altern Med Rev 12, 207-227.

13. Calon F \& Cole G (2007) Neuroprotective action of omega-3 polyunsaturated fatty acids against neurodegenerative diseases: evidence from animal studies. Prostaglandins Leukot Essent Fatty Acids 77, 287-293.

14. Cole GM, Ma QL \& Frautschy SA (2009) Omega-3 fatty acids and dementia. Prostaglandins Leukot Essent Fatty Acids $\mathbf{8 1}$, 213-221.

15. Cunnane SC, Plourde M, Pifferi F, et al. (2009) Fish, docosahexaenoic acid and Alzheimer's disease. Prog Lipid Res $\mathbf{4 8}$, 239-256.

16. Yurko-Mauro K, McCarthy D, Rom D, et al. (2010) Beneficial effects of docosahexaenoic acid on cognition in age-related cognitive decline. Alzheimers Dement 6, 456-464.

17. Bruno MJ, Koeppe RE 2nd \& Andersen OS (2007) Docosahexaenoic acid alters bilayer elastic properties. Proc Natl Acad Sci U S A 104, 9638-9643.

18. Wassall SR \& Stillwell W (2009) Polyunsaturated fatty acidcholesterol interactions: domain formation in membranes. Biochim Biophys Acta 1788, 24-32.

19. Calon F, Lim GP, Yang F, et al. (2004) Docosahexaenoic acid protects from dendritic pathology in an Alzheimer's disease mouse model. Neuron 43, 633-645.

20. Akbar M, Calderon F, Wen Z, et al. (2005) Docosahexaenoic acid: a positive modulator of Akt signaling in neuronal survival. Proc Natl Acad Sci U S A 102, 10858-10863.
21. de Urquiza AM, Liu S, Sjoberg M, et al. (2000) Docosahexaenoic acid, a ligand for the retinoid $\mathrm{X}$ receptor in mouse brain. Science 290, 2140-2144.

22. Ethier I, Kagechika H, Shudo K, et al. (2004) Docosahexaenoic acid reduces haloperidol-induced dyskinesias in mice: involvement of Nur77 and retinoid receptors. Biol Psychiatry 56, 522-526.

23. Calder PC (2002) Dietary modification of inflammation with lipids. Proc Nutr Soc 61, 345-358.

24. Boudrault C, Bazinet RP, Kang JX, et al. (2011) Cyclooxygenase- 2 and $n-6$ PUFA are lower and DHA is higher in the cortex of fat-1 mice. Neurochem Int 56, 585-589.

25. Lalancette-Hébert M, Julien C, Cordeau PJ, et al. (2011) Accumulation of dietary DHA in the brain attenuates acute immune response and development of post-ischemic neuronal damage. Stroke (In the Press).

26. Biou V, Brinkhaus H, Malenka RC, et al. (2008) Interactions between drebrin and Ras regulate dendritic spine plasticity. Eur J Neurosci 27, 2847-2859.

27. Curtis LB, Doneske B, Liu X, et al. (2008) Syntaxin 3b is a t-SNARE specific for ribbon synapses of the retina. J Comp Neurol 510, 550-559.

28. Darios F \& Davletov B (2006) Omega-3 and omega-6 fatty acids stimulate cell membrane expansion by acting on syntaxin 3. Nature 440, 813-817.

29. Cansev M \& Wurtman RJ (2007) Chronic administration of docosahexaenoic acid or eicosapentaenoic acid, but not arachidonic acid, alone or in combination with uridine, increases brain phosphatide and synaptic protein levels in gerbils. Neuroscience 148, 421-431.

30. Arsenault D, Julien C, Tremblay C, et al. (2011) DHA improves cognition and prevents dysfunction of entorhinal cortex neurons in 3xTg-AD mice. PLoS One 6, e17397.

31. Salem N Jr, Pawlosky R, Wegher B, et al. (1999) In vivo conversion of linoleic acid to arachidonic acid in human adults. Prostaglandins Leukot Essent Fatty Acids 60, 407-410.

32. Mayes C, Burdge GC, Bingham A, et al. (2006) Variation in [U-13C] alpha linolenic acid absorption, beta-oxidation and conversion to docosahexaenoic acid in the pre-term infant fed a DHA-enriched formula. Pediatr Res 59, 271-275.

33. Plourde M \& Cunnane SC (2007) Extremely limited synthesis of long chain polyunsaturates in adults: implications for their dietary essentiality and use as supplements. Appl Physiol Nutr Metab 32, 619-634.

34. Burdge GC \& Calder PC (2006) Dietary alpha-linolenic acid and health-related outcomes: a metabolic perspective. Nutr Res Rev 19, 26-52.

35. Jenkins DJ, Sievenpiper JL, Pauly D, et al. (2009) Are dietary recommendations for the use of fish oils sustainable? CMAJ 180, 633-637.

36. Sewards TV \& Sewards MA (2003) Input and output stations of the entorhinal cortex: superficial vs. deep layers or lateral vs. medial divisions? Brain Res Brain Res Rev 42, 243-251.

37. Kumar SS, Jin X, Buckmaster PS, et al. (2007) Recurrent circuits in layer II of medial entorhinal cortex in a model of temporal lobe epilepsy. J Neurosci 27, 1239-1246.

38. Bragin DE, Sanderson JL, Peterson S, et al. (2009) Development of epileptiform excitability in the deep entorhinal cortex after status epilepticus. Eur J Neurosci 30, 611-624.

39. Braak H \& Braak E (1991) Neuropathological stageing of Alzheimer-related changes. Acta Neuropathol 82, 239-259.

40. Gomez-Isla T, Price JL, McKeel DW, et al. (1996) Profound loss of layer II entorhinal cortex neurons occurs in very mild Alzheimer's disease. J Neurosci 16, 4491-4500. 
41. Haroutunian V, Purohit DP, Perl DP, et al. (1999) Neurofibrillary tangles in nondemented elderly subjects and mild Alzheimer disease. Arch Neurol 56, 713-718.

42. Duyckaerts C, Delatour B \& Potier MC (2009) Classification and basic pathology of Alzheimer disease. Acta Neuropathol 118, 5-36.

43. Hogan SA, O'Riordan ED \& O'Sullivan M (2003) Microencapsulation and oxidative stability of spray-dried fish oil emulsions. J Microencapsul 20, 675-688.

44. Kolanowski W, Laufenberg G \& Kunz B (2004) Fish oil stabilisation by microencapsulation with modified cellulose. Int J Food Sci Nutr 55, 333-343.

45. Whelan J \& Rust C (2006) Innovative dietary sources of $n-3$ fatty acids. Annu Rev Nutr 26, 75-103.

46. Diau GY, Hsieh AT, Sarkadi-Nagy EA, et al. (2005) The influence of long chain polyunsaturate supplementation on docosahexaenoic acid and arachidonic acid in baboon neonate central nervous system. BMC Med 3, 11.

47. Brenna JT \& Diau GY (2007) The influence of dietary docosahexaenoic acid and arachidonic acid on central nervous system polyunsaturated fatty acid composition. Prostaglandins Leukot Essent Fatty Acids 77, 247-250.

48. Dullemeijer C, Zock PL, Coronel R, et al. (2008) Differences in fatty acid composition between cerebral brain lobes in juvenile pigs after fish oil feeding. Br J Nutr 100, 794-800.

49. Hsieh AT \& Brenna JT (2009) Dietary docosahexaenoic acid but not arachidonic acid influences central nervous system fatty acid status in baboon neonates. Prostaglandins Leukot Essent Fatty Acids 81, 105-110.

50. Julien C, Berthiaume L, Hadj-Tahar A, et al. (2006) Postmortem brain fatty acid profile of levodopa-treated Parkinson disease patients and parkinsonian monkeys. Neurochem Int 48, 404-414.

51. Folch J, Lees M \& Sloane Stanley GH (1957) A simple method for the isolation and purification of total lipides from animal tissues. J Biol Chem 226, 497-509.

52. Zhang ZW \& Arsenault D (2005) Gain modulation by serotonin in pyramidal neurones of the rat prefrontal cortex. $J$ Physiol 566, 379-394.

53. Arsenault D \& Zhang ZW (2006) Developmental remodelling of the lemniscal synapse in the ventral basal thalamus of the mouse. J Physiol 573, 121-132.

54. Golowasch J, Thomas G, Taylor AL, et al. (2009) Membrane capacitance measurements revisited: dependence of capacitance value on measurement method in nonisopotential neurons. J Neurophysiol 102, 2161-2175.

55. Bourre JM, Francois M, Youyou A, et al. (1989) The effects of dietary alpha-linolenic acid on the composition of nerve membranes, enzymatic activity, amplitude of electrophysiological parameters, resistance to poisons and performance of learning tasks in rats. J Nutr 119, 1880-1892.

56. Salem N Jr, Moriguchi T, Greiner RS, et al. (2001) Alterations in brain function after loss of docosahexaenoate due to dietary restriction of $n-3$ fatty acids. J Mol Neurosci 16, 299-307, (discussion 317-221).

57. Green KN, Martinez-Coria H, Khashwji H, et al. (2007) Dietary docosahexaenoic acid and docosapentaenoic acid ameliorate amyloid-beta and tau pathology via a mechanism involving presenilin 1 levels. J Neurosci 27, 4385-4395.

58. Julien C, Tremblay C, Phivilay A, et al. (2010) High-fat diet aggravates amyloid-beta and tau pathologies in the $3 \times \mathrm{Tg}$ AD mouse model. Neurobiol Aging 31, 1516-1531.

59. Gentet LJ, Stuart GJ \& Clements JD (2000) Direct measurement of specific membrane capacitance in neurons. Biophys $J$ 79, 314-320.
60. Faumont S, Boulin T, Hobert O, et al. (2006) Developmental regulation of whole cell capacitance and membrane current in identified interneurons in C. elegans. J Neurophysiol $\mathbf{9 5}$, 3665-3673

61. Robson LG, Dyall S, Sidloff D, et al. (2008) Omega-3 polyunsaturated fatty acids increase the neurite outgrowth of rat sensory neurones throughout development and in aged animals. Neurobiol Aging 31, 678-687.

62. Cao D, Kevala K, Kim J, et al. (2009) Docosahexaenoic acid promotes hippocampal neuronal development and synaptic function. J Neurochem 111, 510-521.

63. He C, Qu X, Cui L, et al. (2009) Improved spatial learning performance of fat-1 mice is associated with enhanced neurogenesis and neuritogenesis by docosahexaenoic acid. Proc Natl Acad Sci U S A 106, 11370-11375.

64. Shim KS \& Lubec G (2002) Drebrin, a dendritic spine protein, is manifold decreased in brains of patients with Alzheimer's disease and Down syndrome. Neurosci Lett 324, 209-212.

65. Sekino Y, Kojima N \& Shirao T (2007) Role of actin cytoskeleton in dendritic spine morphogenesis. Neurochem Int 51, 92-104.

66. Moolman DL, Vitolo OV, Vonsattel JP, et al. (2004) Dendrite and dendritic spine alterations in Alzheimer models. J Neurocytol 33, 377-387.

67. Ouellet M, Emond V, Chen CT, et al. (2009) Diffusion of docosahexaenoic and eicosapentaenoic acids through the blood-brain barrier: an in situ cerebral perfusion study. Neurochem Int 55, 476-482.

68. Rapoport SI, Rao JS \& Igarashi M (2007) Brain metabolism of nutritionally essential polyunsaturated fatty acids depends on both the diet and the liver. Prostaglandins Leukot Essent Fatty Acids 77, 251-261.

69. Mohrhauer H \& Holman RT (1963) Alteration of the fatty acid composition of brain lipids by varying levels of dietary essential fatty acids. J Neurochem 10, 523-530.

70. Galli C, White HB Jr \& Paoletti R (1971) Lipid alterations and their reversion in the central nervous system of growing rats deficient in essential fatty acids. Lipids 6, 378-387.

71. Bourre JM (1989) Nature, origin and role of fatty acids of the nervous system: an essential fatty acid, an alpha-linolenic acid, changing the structure and the cerebral function. Bull Acad Natl Med 173, 1137-1148, discussion 1148-1151.

72. Brenna JT (2011) Animal studies of the functional consequences of suboptimal polyunsaturated fatty acid status during pregnancy, lactation and early post-natal life. Matern Child Nutr 7, Suppl. 2, 59-79.

73. Abedin L, Lien EL, Vingrys AJ, et al. (1999) The effects of dietary alpha-linolenic acid compared with docosahexaenoic acid on brain, retina, liver, and heart in the guinea pig. Lipids 34, 475-482.

74. Youyou A, Durand G, Pascal G, et al. (1986) Recovery of altered fatty acid composition induced by a diet devoid of $n-3$ fatty acids in myelin, synaptosomes, mitochondria, and microsomes of developing rat brain. I Neurochem $\mathbf{4 6}$, $224-228$.

75. Groeger AL, Cipollina C, Cole MP, et al. (2011) Cyclooxygenase- 2 generates anti-inflammatory mediators from omega- 3 fatty acids. Nat Chem Biol 6, 433-441.

76. Wurtman RJ, Cansev M \& Ulus IH (2009) Synapse formation is enhanced by oral administration of uridine and DHA, the circulating precursors of brain phosphatides. $J$ Nutr Health Aging 13, 189-197.

77. Sakamoto T, Cansev M \& Wurtman RJ (2007) Oral supplementation with docosahexaenoic acid and uridine- $5^{\prime}$ - 
monophosphate increases dendritic spine density in adult gerbil hippocampus. Brain Res 1182, 50-59.

78. Susarla BT, Seal RP, Zelenaia O, et al. (2004) Differential regulation of GLAST immunoreactivity and activity by protein kinase $\mathrm{C}$ : evidence for modification of amino and carboxyl termini. J Neurochem 91, 1151-1163.

79. Tilleux S \& Hermans E (2008) Down-regulation of astrocytic GLAST by microglia-related inflammation is abrogated in dibutyryl cAMP-differentiated cultures. I Neurochem 105 , $2224-2236$

80. Grintal B, Champeil-Potokar G, Lavialle M, et al. (2009) Inhibition of astroglial glutamate transport by polyunsaturated fatty acids: evidence for a signalling role of docosahexaenoic acid. Neurochem Int 54, 535-543.

81. Robinson MB (2006) Acute regulation of sodium-dependent glutamate transporters: a focus on constitutive and regulated trafficking. Handb Exp Pharmacol 251-275.

82. Chytrova G, Ying Z \& Gomez-Pinilla F (2010) Exercise contributes to the effects of DHA dietary supplementation by acting on membrane-related synaptic systems. Brain Res 1341, 32-40.

83. Kojima N, Hanamura K, Yamazaki H, et al. (2010) Genetic disruption of the alternative splicing of drebrin gene impairs context-dependent fear learning in adulthood. Neuroscience 165, 138-150.

84. Toda M, Shirao T \& Uyemura K (1999) Suppression of an actin-binding protein, drebrin, by antisense transfection attenuates neurite outgrowth in neuroblastoma B104 cells. Brain Res Dev Brain Res 114, 193-200.

85. Catalan J, Moriguchi T, Slotnick B, et al. (2002) Cognitive deficits in docosahexaenoic acid-deficient rats. Behav Neurosci 116, 1022-1031.

86. Petursdottir AL, Farr SA, Morley JE, et al. (2008) Effect of dietary $n-3$ polyunsaturated fatty acids on brain lipid fatty acid composition, learning ability, and memory of senescence-accelerated mouse. J Gerontol A Biol Sci Med Sci 63 $1153-1160$.

87. Yurko-Mauro K (2010) Cognitive and cardiovascular benefits of docosahexaenoic acid in aging and cognitive decline Curr Alzheimer Res 7, 190-196.

88. Cole GM \& Frautschy SA (2010) DHA may prevent agerelated dementia. J Nutr 140, 869-874.

89. Fedorova I, Alvheim AR, Hussein N, et al. (2009) Deficit in prepulse inhibition in mice caused by dietary $n$-3 fatty acid deficiency. Behav Neurosci 123, 1218-1225.

90. Lafourcade M, Larrieu T, Mato S, et al. (2011) Nutritional omega-3 deficiency abolishes endocannabinoid-mediated neuronal functions. Nat Neurosci 14, 345-350.

91. Blondeau N, Nguemeni C, Debruyne DN, et al. (2009) Subchronic alpha-linolenic acid treatment enhances brain plasticity and exerts an antidepressant effect: a versatile potential therapy for stroke. Neuropsychopharmacology 34, $2548-2559$.

92. Xiao Y \& Li X (1999) Polyunsaturated fatty acids modify mouse hippocampal neuronal excitability during excitotoxic or convulsant stimulation. Brain Res 846, 112-121.

93. Young C, Gean PW, Chiou LC, et al. (2000) Docosahexaenoic acid inhibits synaptic transmission and epileptiform activity in the rat hippocampus. Synapse 37, 90-94.

94. Taha AY, Huot PS, Reza-Lopez S, et al. (2008) Seizure resistance in fat-1 transgenic mice endogenously synthesizing high levels of omega-3 polyunsaturated fatty acids. J Neurochem 105, 380-388.

95. Taha AY, Burnham WM \& Auvin S (2010) Polyunsaturated fatty acids and epilepsy. Epilepsia 51, 1348-1358.

96. Schlanger S, Shinitzky M \& Yam D (2002) Diet enriched with omega-3 fatty acids alleviates convulsion symptoms in epilepsy patients. Epilepsia 43, 103-104. 